\title{
Ensino em serviço na perspectiva da Clínica Ampliada: relato de experiência
}

\author{
Osmar José da Silva Júnior*; Flávio Renato Reis de Moura**; Raul Antônio Cruz**; Myrian Camara \\ Brew**; Caren Serra Bavaresco** $^{* *}$ \\ * Acadêmico do curso de Odontologia da Universidade \\ Luterana do Brasil, ULBRA Canoas \\ ** Docente do curso de Odontologia da Universidade Luterana \\ do Brasil, ULBRA Canoas
}

Recebido em 26/07/2017. Aprovado em 17/11/2017.

\begin{abstract}
RESUMO
A Clínica Ampliada pode ser compreendida como a relação estabelecida entre os diferentes profissionais de saúde com os usuários no intuito de produzir saúde por meio do empoderamento dos sujeitos e criação de autonomia. Neste contexto, a formação profissional em Odontologia deve primar pelo desenvolvimento das habilidades técnicas aliadas a qualidades humanistas para atuação na perspectiva da Clínica Ampliada. Considerando a discussão do processo de formação do cirurgiãodentista integrado ao serviço de saúde pública e a perspectiva de atuação na Clínica Ampliada, este trabalho teve como objetivo relatar a experiência acadêmica do estágio supervisionado, realizado um uma unidade de saúde, no município de Porto Alegre/RS. No modelo de um relato de experiência descritivo, com metodologia relacionada ao processo reflexivo crítico do aluno, o estágio ocorreu num período de 30 dias, com carga horária de 15 horas semanais, em uma unidade de saúde pertencente ao Grupo Hospitalar Conceição. Incluiu atividades de acompanhamento ambulatorial e de promoção e prevenção de saúde, realizados na unidade de saúde e sua área de atuação. Da experiência vivida, foi possível observar que os profissionais da equipe de saúde tinham como proposta para o atendimento a resolutividade clínica e a perspectiva de um plano de tratamento conjunto, de acordo com a realidade social de cada usuário, baseando-se na comunicação horizontal entre comunidade e profissionais. Assim, compreender a saúde bucal dentro da perspectiva da Clínica Ampliada requer, cada vez mais, uma análise conjunta das questões biopsicossociais dos indivíduos. Descritores: Educação em Odontologia. Atenção Primária à Saúde. Saúde Bucal.
\end{abstract}

\section{INTRODUÇÃO}

O ensino da Odontologia, na perspectiva da Clínica Ampliada, necessita da compreensão de fatores como a organização dos serviços de saúde e as condições sociodemográficas, culturais e de saúde da população. Ou seja, o conceito ampliado de saúde tende a se fusionar com a proposta de Clínica Ampliada. Portanto, é vital o desdobramento do ensino-serviço na área da
Odontologia, compreendendo a estruturação em bases políticas, éticas e científicas.

Embora tenham ocorrido movimentos significativos para uma reflexão crítica sobre os modelos tradicionais de formação profissional na saúde, em relação à Odontologia existe um atraso histórico desses movimentos de mudança, exigindo um esforço redobrado para que se possa integrar a saúde bucal dentro do novo contexto 
de ação interdisciplinar e multiprofissional ${ }^{1}$.

$\mathrm{Na}$ Odontologia, vale ressaltar a importância da inserção precoce do graduando no contexto profissional da Atenção Primária à Saúde (APS) com intuito de melhorar a formação em Saúde Coletiva. Além disso, sugere-se a diversificação dos cenários de práticas $^{2}$, como a inserção dos graduandos em atividades da atenção primária, secundária e terciária em saúde, levando à compreensão das redes de atenção As Diretrizes Curriculares Nacionais (DCN) afirmam que "o Estágio Supervisionado (ES) é o instrumento de integração e conhecimento do aluno em relação a realidade social e econômica de sua região e do trabalho de sua área, podendo ser realizado por meio de atendimentos multidisciplinares e em serviços assistenciais públicos e privados" ${ }^{\text {"-5 }}$.

Em termos de formação profissional, é necessário capacitar, formar e educar permanentemente os profissionais de saúde bucal atuantes na APS por intermédio da articulação entre as instituições de ensino superior e os serviços vinculados ao Sistema Único de Saúde (SUS), bem como fomentar as habilidades individuais e coletivas relacionadas à avaliação dos padrões de qualidade e do impacto das ações de saúde bucal desenvolvidas, de acordo com os princípios da Estratégia Saúde da Família $(\mathrm{ESF})^{6-8}$.

A Odontologia, por meio de seus atores e atribuições, deve ser incluída nessa modalidade de atenção à saúde regida pelos princípios da APS, cultivando uma relação nova com a comunidade, baseada na atenção, na confiança, no respeito e no cuidado ${ }^{8}$. Com a implantação da Política Nacional de Saúde Bucal (PNSB) foi possível estabelecer subsídios nacionais do processo de trabalho das equipes de saúde bucal, definindo parâmetros em relação às ações individuais e coletivas no que tange a prevenção, promoção de saúde e reabilitação da saúde bucal no país. A PNSB preconiza a criação de linhas de cuidado, o estabelecimento de redes de atenção à saúde e o trabalho baseado na concepção de clínica ampliada, quebrando os conceitos verticalizados da atenção odontológica historicamente prestada no Brasil ${ }^{9}$. A Clínica Ampliada, segundo preconizado pelo Ministério da Saúde, refere-se à relação estabelecida entre os diferentes profissionais de saúde com os usuários com o intuito de produzir saúde pelo empoderamento dos sujeitos e de autonomia. $\mathrm{O}$ trabalho na Clínica Ampliada pressupõe um modo de agir em saúde centrado no usuário, e que possa ir para além do processo saúde-doença, favorecendo desfechos mais positivos na saúde ${ }^{10}$.

Considerando a discussão do processo de formação do cirurgião-dentista integrado ao serviço de saúde pública na perspectiva da clínica ampliada, este trabalho teve como objetivo relatar a experiência acadêmica de um graduando em estágio supervisionado eletivo voluntário realizado em uma unidade do Grupo Hospitalar Conceição (GHC) no município de Porto Alegre, RS.

\section{RELATO DE EXPERIÊNCIA}

Descreve-se a vivência de um graduando do décimo semestre do Curso de Odontologia em estágio eletivo voluntário com duração de um mês (entre novembro e dezembro de 2016), com carga horária de 15 horas semanais, em uma Unidade Básica de Saúde pertencente ao GHC.

O GHC mantém o Serviço de Saúde Comunitária (SSC) com 12 unidades de atenção primária e atua como centro formador para o SUS, sendo um reconhecido programa de residência em medicina de família e comunidade, além da residência integrada em saúde multiprofissional, considerando os princípios da Clínica Ampliada. Nestas unidades básicas ocorre a capacitação de equipes multiprofissionais, treinamento de equipes de ESF, desenvolvimento de protocolos clínicos 
para a rede básica de saúde, produção de promoção e educação em saúde, atendimento domiciliar e, ainda, estágios curriculares para diversos cursos, de nível superior ou técnico.

A escolha pelo local do estágio baseou-se no reconhecimento do GHC como referência na formação de profissionais para a APS. O estágio voluntário incluiu atividades de acompanhamento ambulatorial e de promoção de saúde e prevenção de doenças, realizados na Unidade Básica de Saúde, incluindo grupos de educação em saúde, atuação em áreas de vigilância e acolhimento ${ }^{11}$. O percurso do aluno ocorreu de forma sistematizada a fim de aproveitar as ações realizadas dentro da unidade de saúde e na comunidade durante período do estágio.

A unidade de saúde ${ }^{12}$ caracteriza-se como equipe parametrizada, compreendida como a equipe de atenção básica tradicional que foi padronizada em relação ao tipo de profissional, carga horária e adscrição da população.

O processo de trabalho da equipe está pautado nas ações de promoção de saúde, prevenção e recuperação dos agravos, conforme pautado pela PNAB $^{13}$ e nos processos da clínica ampliada. A unidade está organizada para acolher os usuários em suas demandas programadas e espontâneas, dentro do conceito de episódio de cuidado. Através da escuta qualificada, o profissional da saúde busca compreender os motivos pelos quais o usuário adoeceu ou buscou atendimento, como está se sentindo e quais são os seus sintomas, a fim de atuar na corresponsabilização do cuidado e na construção de práticas saudáveis.

O acesso está organizado de forma agendada - para as consultas programadas, e abertas - para as consultas do dia. Os usuários possuem à sua disposição as agendas dos profissionais com até 3 meses de antecedência. São consideradas prioridades de acesso as gestantes, crianças até 5 anos, hipertensos, diabéticos e idosos.

A vigilância em saúde realiza ações de educação e promoção de saúde, além de planejar e executar o acompanhamento da população, reconhecer o território, monitorar e desenvolver ações programáticas e levantar situações e famílias em vulnerabilidade ${ }^{13}$.

Quanto ao ambulatório de Odontologia, sua estrutura física é composta pelo equipamento odontológico, instrumental, mobiliário e material de escritório. As atividades são desenvolvidas com a participação de dois cirurgiões-dentistas e dois técnicos de saúde bucal, com tempo médio de 30 minutos para atendimento.

Durante o estágio supervisionado (ES), foi possível conhecer as normatizações da Equipe de Saúde Bucal (ESB) na ESF - ampliação do acesso, melhoria das condições de saúde bucal da população brasileira e orientação das práticas de atenção à saúde bucal. Além disso, foi possível compreender que a equipe deve assegurar o acesso progressivo de todas as famílias residentes nas áreas cobertas pelas ESF, ofertando ações de promoção e de prevenção, bem como aquelas de caráter curativorestauradoras de saúde bucal.

Os profissionais da Equipe de Saúde Bucal (ESB) tinham como proposta para o atendimento da população, além da resolutividade clínica, a perspectiva de um plano de tratamento conjunto, compreendendo a necessidade de um plano terapêutico singular e compartilhado, baseado na comunicação transversal, além de atuarem como gestores dos casos dentro da equipe, o que permite a compreensão da atuação do cirurgião-dentista inserido na Clínica Ampliada.

Sobre a experiência do graduando, pode-se dizer que um estranhamento inicial decorrente do processo assistencial permeado pelas limitações dos recursos materiais foi sendo substituído por 
um sentimento de satisfação a cada novo atendimento. Perceber que a atuação odontológica não está engessada em um modelo de atuação baseado exclusivamente em tecnologias duras proporcionou uma nova reflexão sobre o fazer em saúde.

O acolhimento das demandas em Saúde Bucal é realizado pela ESB, como forma de tornar mais dinâmica a resolução do problema de saúde de cada usuário e compõem uma interligação de espaços que visam a escuta qualificada e flexibilização da atenção. $\mathrm{O}$ dia a dia demonstrou que a equipe incorpora os princípios destacados por Starfield ${ }^{14,15}$ durante seu processo trabalho, como o acesso, a integralidade, a longitudinalidade e a coordenação do cuidado, bem como a abordagem familiar e comunitária e a competência cultural fazem parte de seus atributos derivados ${ }^{15}$.

As discussões sobre acesso foram incansáveis durante o ES e foi possível perceber que o acolhimento é uma forma de garantia do acesso nos casos de demanda espontânea ${ }^{16}$. Todos os pacientes que residem na área adscrita à unidade de saúde recebem atendimento humanizado e centrado nas suas necessidades. Neste contexto, o processo de trabalho desenvolvido pela equipe proporciona a abertura para a escuta do outro, além de facilitar a interação e o vínculo do estudante com os atores envolvidos neste processo (comunidade, equipe e universidade), visando um cuidado integral, integrado e humanizado.

A vivência demonstrou bom vínculo entre a equipe de saúde e a população atendida. A equipe preza pelo vínculo dos profissionais com usuários, os quais serão, preferencialmente, atendidos pelo mesmo profissional (médico, psicólogo/assistente social, enfermeiro ou cirurgião-dentista) responsável pela área de vigilância a qual o paciente pertence ${ }^{18}$. A formação de vínculo foi uma das potencialidades observadas durante o ES, o que contribui para o fortalecimento dos princípios da APS, por exemplo, tornando possível o cuidado longitudinal.

Por observação, verificou-se que os usuários demonstravam satisfação com os serviços prestados pela unidade de saúde e eram muito agradecidos aos profissionais que os atendiam.

Neste estágio, foi possível observar a existência de um modelo assistencial consolidado e ciente de seu papel para com a comunidade. Poder vivenciar a APS e a troca de informações com enfermeiros, psicólogos, médicos, técnicos em saúde bucal, agentes comunitários e cirurgiões-dentistas, foi muito valioso e enriquecedor como estratégia de consolidação da clínica ampliada fortalecida pela atenção à saúde de maneira multiprofissional. Além disso, o estagiário conseguiu estabelecer um vínculo de amizade com a equipe, facilitando o processo de ensino-aprendizagem em serviço.

Nas DCN, a preocupação com a formação voltada para o SUS consta no item XXIX das habilidades específicas para Odontologia - no qual se afirma que o curso deve capacitar o aluno para "planejar e administrar serviços de saúde comunitária". No parágrafo único do artigo $5^{\circ}$, destaca-se a necessidade da formação para o SUS, compreendendo as dinâmicas das redes de atenção à saúde, do processo de trabalho e das diretrizes da $\mathrm{PNAB}^{13}$, diretrizes materializadas e vivenciadas durante o estágio executado,

Ayers et al. $^{22}$ defenderam que as atividades extramuros deveriam ser vistas como componentes importantes de qualquer currículo de Odontologia. O conceito sobre estágio para Odontologia foi desenvolvido em reuniões da Associação Brasileira de Ensino Odontológico (ABENO) e pode ser descrito como instrumento de formação e integração do estudante com a realidade social e econômica ${ }^{21}$. Sem dúvida, a 
inserção do acadêmico desde o início do curso nos serviços de saúde contribuirá para um atendimento mais humanizado e completo ao longo da formação em saúde.

Todavia, apesar de ser uma unidade de saúde bem estruturada e com profissionais engajados com as necessidades da população local, foi possível perceber que a grande demanda torna alguns encaminhamentos ou planos de tratamento de difícil acesso, levando em consideração a disparidade do tempo do cuidado com o tempo de espera para a resolução da demanda. Espera-se que, com a qualificação cada vez maior da rede de atenção à saúde e dos profissionais que a constituem, seja possível ultrapassar tais barreiras.

Outro ponto a ser destacado refere-se à infraestrutura que, muitas vezes, não estava totalmente adequada às necessidades da equipe $\mathrm{e}$ da população. Há necessidade de compra de insumos e equipamentos, melhorias na segurança e iluminação pública ao redor da unidade de saúde. Cabe ressaltar que este relato se refere à percepção de apenas um aluno no contexto atual da equipe, podendo apresentar configuração diferente em outro período de análise.

\section{CONSIDERAÇÕES FINAIS}

A Odontologia, como profissão da área da saúde, visa o bem-estar e as condições para tal, contando com uma gama infinita de recursos e metodologias, como também com aparatos tecnológicos, instrumentos e produtos, que vão desde a estética até procedimentos que têm a capacidade de devolver, não somente a qualidade de vida, mas a dignidade humana.

Compreender a saúde bucal dentro da perspectiva da clínica ampliada requer, cada vez mais, uma análise conjunta das questões biopsicosociais dos indivíduos. Afinal, faz-se necessário a mudança da visão de "Odontologia de posto" para a real complexidade de atender indivíduos de forma integral em um campo de atuação tão rico.

\section{ABSTRACT \\ Education-service at the Expanded Clinic perspective: an experience report}

The expanded clinic can be understood as the relationship established between the different health professionals with the users in order to produce health through the empowerment of the subjects and creation of autonomy. In this context, professional training in dentistry should be based on the development of technical skills combined with humanistic qualities to act in the perspective of the Expanded Clinic. Considering the debate regarding the formation of dentists integrated into the public health service and considering the prospect of performance in the expanded clinic, the purpose of this study is to report the academic experience of a supervised internship, in a health unit, in Porto Alegre/RS. The internship took place over a thirty-day period, with a workload of 15 hours per week, at the one health unit of the Grupo Hospitalar Conceição, and it was structured on a descriptive experience report method, related to the student's critical reflective process. The internship included activities of outpatient follow-up and health promotion and prevention, performed at the health unit and its operating fields. From the experience it was possible to observe that the team's proposal for the service performance it the conclusion of the treatment and the perspective of a joint treatment plan, specific to the social reality of each patient, based on the horizontal communication between the community and the professionals. Finally, understanding oral health within the perspective of the extended clinic requires a joint analysis of the bio-psycho-social issues of the persons.

Descriptors: Education, Dental. Primary Health Care. Oral Health.

\section{REFERÊNCIAS}

1. Finkler M, Caetano JC, Ramos FR. Integração "ensino-serviço" no processo de 
mudança na formação profissional em Odontologia. Interface. 2011;15(39):105370 .

2. Morita MC, Kriger L. Mudanças nos cursos de Odontologia e a interação com o SUS. Rev ABENO. 2004;4(1):17-21.

3. Warmling CM, Marzola NR, Botazzo C. Da autonomia da boca: práticas curriculares e identidade profissional na emergência do ensino brasileiro da odontologia. Hist Ciênc Saúde. 2012 jan/mar;19(1):181-95.

4. Brasil. Diretrizes Curriculares Nacionais dos Cursos de Graduação em Farmácia e Odontologia. Parecer CNE/CES n $1.300 / 01$, aprovado em 6 de novembro de 2001. [Acesso em 21 set. 2017]. Disponível em: http://portal.mec.gov.br/educacao-quilombo la-/323-secretarias-112877938/orgaos-vincu lados-82187207/12991-diretrizes-curricular es-cursos-de-graduacao.

5. Associação Brasileira de Ensino Odontológico (ABENO). Diretrizes da ABENO para a definição do estágio supervisionado nos cursos de Odontologia. Rev ABENO. 2002;2(1):13-8.

6. Faé JM, Silva Junior MF, Carvalho RB, Esposti CDD, Pacheco KTS. A integração ensino-serviço em Odontologia no Brasil. Rev ABENO. 2016;16(3):7-18.

7. Brasil. Portaria $n^{\circ} 267$, de 06 de março de 2001. Aprova as normas e diretrizes de inclusão da saúde bucal na estratégia do Programa de Saúde da Família (PSF). Diário Oficial da República Federativa do Brasil. 2001 mar; Seção1. p. 67.

8. Celeste RK, Moura FR. Programa de saúde da família em Cachoeira do Sul: a inclusão de acadêmicos de odontologia. Odontologia e Sociedade. 2006;9(1):10.

9. Brasil. Ministério da Saúde. Diretrizes da política nacional de saúde bucal. Brasília: Ministério da Saúde: 2004.
10. Brasil. Ministério da Saúde. Secretaria de Atenção à Saúde. Política Nacional de Humanização. Formação e intervenção / Ministério da Saúde, Secretaria de Atenção à Saúde, Política Nacional de Humanização. Brasília : Ministério da Saúde; 2010.

11. Brasil. Ministério da Saúde. Departamento de atenção básica. Guia prático do Programa de Saúde da Família. Brasília: Ministério da Saúde; 2001.

12. Martini JG. Implantação do Programa de Saúde da Família em Porto Alegre. R Bras Enferm. 2000;53:71-6.

13. Brasil. Ministério da Saúde. Portaria $n^{\circ}$ 2.436 de 21 de setembro de 2017. Política Nacional de Atenção Básica. Diário Oficial da República Federativa do Brasil. 2017 set; Seção 1. n. 183.

14. Giovanella L, Mendonça MHM, Almeida PF, Escorel Sarah, Senna MCM, Fausto MCC et al. Saúde da família: limites e possibilidades para uma abordagem integral de atenção primária à saúde no Brasil. Ciênc Saúde Colet. 2009;14(3):783-94.

15. Amoretti R. A Educação Médica diante das necessidades sociais em saúde. Rev Bras Educ Méd.2005;29(2):136-46.

16. Starfield B. Primary Care: concept, evaluations, and policy. Oxford University Press, New York. 1992.

17. Starfield B. Atenção primária: equilíbrio entre necessidades de saúde, serviços e tecnologia. Brasília: Unesco/Ministério da Saúde; 2002.

18. Paim JS. Atenção à saúde no Brasil. In: Ministério da Saúde. Saúde no Brasil Contribuições para a agenda de prioridades de pesquisa. Brasília (DF); 2004. p. 15-44.

19. Falk MLR, Falk JW, Oliveira FA, Motta MS. Acolhimento como dispositivo de humanização: percepção do usuário e do trabalhador em saúde. Rev APS. 2010; 
13(1):4-9.

20. Camelo SHH, Angerami ELS, Silva EM, Mishima SM. Acolhimento clientela: estudo em unidades básicas de saúde no município de Ribeirão Preto. Rev Latinoam Enferm. 2000;8(4):30-7.

21. Brasil. Ministério da Educação. Conselho Nacional de Educação. Câmara de Educação Superior. Diretrizes Curriculares Nacionais do Curso de Graduação em Odontologia. Diário Oficial da União, Brasília, DF, 4 mar. 2002. n. 4, Seção. 1, p. 10.

22. Ayers CS, Abrams RA, McCunniff MD, Goldstein BR. A comparison of private and public dental students' perceptions of extramural programming. $\mathrm{J}$ Dent Educ. 2003;67(4):412-7.

23. Carvalho ACP. Ensino em Odontologia no Brasil. In: Perri de Carvalho AC, Kriger L. Educação Odontológica. São Paulo: Artes Médicas, 2006. p. 5-15.

Correspondência para:

Caren Serra Bavaresco

e-mail: c_bavaresco@yahoo.com.br Av. Farroupilha, 8001 Prédio 59

92424-900 Igara - Canoas/ RS 